\title{
Alteraciones auditivas en neonatos pretérmino a su egreso de una unidad de cuidados intensivos neonatales
}

\section{Hearing disorders in preterm neonates upon leaving a neonatal intensive care unit}

Andrea Venegas-Andrade, ${ }^{1}$ Carlos Armando Tello-Valdés, ${ }^{2}$ José Iglesias-Leboreiro, ${ }^{3}$ Isabel Bernárdez-Zapata, ${ }^{4}$ Ana Paula Cuevas-Rivas, ${ }^{5}$ Paola Rodríguez-Santaolaya, ${ }^{1}$ Irma Gabriela De Luna-Sánchez, ${ }^{5}$ Celia Monserratte Ortega-Cisneros ${ }^{1}$

\begin{abstract}
Resumen
OBJETIVO: Estimar la frecuencia de alteraciones auditivas en neonatos pretérmino a su egreso de la unidad de cuidados intensivos neonatales e identificar los factores de riesgo asociados.

MATERIALES Y MÉTODOS: Estudio transversal y retrospectivo efectuado en pacientes prematuros nacidos en el Hospital Español de México entre 2014 y 2016. Variables estudiadas: género, semanas de gestación al nacimiento, alteraciones neonatales y exposición a dos medicamentos ototóxicos. Se valoraron los potenciales evocados auditivos del tallo cerebral (PEATC) en las 72 horas previas a su egreso de cuidados intensivos. Los riesgos se expresan con razón de momios (OR).

RESULTADOS: Se incluyeron 364 neonatos: $53 \%$ hombres y $47 \%$ mujeres; nacidos a las 34 semanas de gestación (mediana). Se encontró restricción del crecimiento intrauterino en $12.9 \%$, hiperbilirrubinemia $49.7 \%$, sepsis $31.0 \%$, hemorragia intraventricular $3.6 \%$, encefalopatía hipóxico isquémica $1.4 \%$, exposición a furosemida $11.0 \%$ y a aminoglucósidos $64.6 \%$. Las alteraciones encontradas en los PEATC fueron: alargamiento de la latencia interpico I-III en $37.9 \%$, III-V en $4.4 \%$ y I-V en $13.5 \%$. La onda I fue anormal en $6.6 \%$, III en $1.9 \%$ y $\mathrm{V}$ en $10.4 \%$ de todos los casos.

CONCLUSIONES: Se descubrieron cuatro factores de riesgo: hiperbilirrubinemia, sepsis, hemorragia intraventricular y el uso de furosemida, que incrementaron la probabilidad de sufrir defectos en la conducción auditiva en las latencias interpico I-III, III-V y I-V. La restricción del crecimiento intrauterino fue un factor protector para alteraciones auditivas a nivel central.
\end{abstract}

PALABRAS CLAVE: Potenciales evocados auditivos; pretérmino; riesgo; alteraciones auditivas.

Abstract

OBJECTIVE: To estimate the frequency of hearing disorders of preterm infants upon leaving from the neonatal intensive care unit (NICU), as well as to identify the risk factors associated with these abnormalities.

MATERIAL AND METHODS: Retrospective cross-sectional study with pre-term infants born at the Hospital Español between 2014 and 2016. Sex, gestational age at birth, various neonatal pathologies and exposure to two ototoxic drugs were evaluated. All subjects were evaluated with ABRs within 72 hours prior leaving NICU. The risks were presented as odds ratio (OR).

RESULTS: 364 neonates were included, with gestational age at birth of 34 weeks (median), 53\% male and $47 \%$ female. They presented intrauterine growth restriction $12.9 \%$, hyperbilirubinemia $49.7 \%$, sepsis $31.0 \%$, intraventricular hemorrhage $3.6 \%$,

\author{
${ }^{1}$ Residente de Pediatría, Facultad \\ Mexicana de Medicina, Universidad La \\ Salle, México. \\ ${ }^{2}$ Neurofisiólogo clínico. \\ 3 Pediatra neonatólogo, jefe de la \\ División de Pediatría y de la Unidad \\ de Cuidados Intensivos Neonatales, \\ profesor titular del Curso de Pediatría \\ y Neonatología. \\ ${ }^{4}$ Pediatra neonatóloga, jefa del Cunero \\ Fisiológico y profesora del Curso de \\ Pediatría y Neonaología. \\ ${ }^{5}$ Pediatra. \\ Hospital Español de México, Ciudad \\ de México. \\ Recibido: 5 de julio 2019
}

Aceptado: 28 de noviembre de 2019

Correspondencia

Andrea Venegas Andrade

dravenegas.ava@gmail.com

Este artículo debe citarse como Venegas-Andrade A, Tello-Valdés CA, Iglesias-Leboreiro J, Bernárdez-Zapata I, Cuevas-Rivas AP, Rodríguez-Santaolaya P, De Luna-Sánchez IG, Ortega-Cisneros $\mathrm{CM}$. Alteraciones auditivas en neonatos pretérmino a su egreso de una unidad de cuidados intensivos neonatales. Acta Pediatr Mex. 2020;41(1):1-10. 
ischemic hypoxic encephalopathy $1.4 \%$, furosemide exposure $11.0 \%$ and aminoglycosides $64.6 \%$. ABR abnormalities were as follows: increments of interpeak latency I-III $37.9 \%$, III-V $4.4 \%$ and I-V $13.5 \%$. Wave I latencies were prolonged in $6.6 \%$, wave III in $1.9 \%$ and $10.4 \%$ of wave $\mathrm{V}$.

CONCLUSIONS: In accordance to previous studies, we found that hyperbilirubinemia, sepsis, intraventricular hemorrhage and furosemide use in premature babies, increase the probability of auditory conduction abnormalities in interpeak latencies I-III, III-V and I-V. Intrauterine growth restriction was a protective factor in this sample.

KEYWORDS: Auditory evoked potentials; Pre term; Risk; Hearing disorders.

\section{ANTECEDENTES}

El déficit auditivo es un problema común en la infancia, con una incidencia que varía de 0.1 $0.6 \%$ en neonatos sanos en todo el mundo. ${ }^{1}$ Esta cifra se incrementa de $2-4 \%$ en egresados de la unidad de cuidados intensivos neonatales (UCIN), incluso a $10 \%$ en prematuros. ${ }^{1}$ Algunos estudios evidencian la asociación entre audición alterada y problemas en el desarrollo del habla y el lenguaje $y$, por ende, en el área cognitiva y conductual durante la infancia. ${ }^{2}$ Por lo anterior, actualmente varios autores recomiendan la valoración del sistema auditivo a través de los potenciales evocados auditivos del tallo cerebral (PEATC) en todo neonato con estancia mayor de cinco días en la UCIN. ${ }^{1,3}$ Los PEATC son la prueba idónea para valorar la maduración de la vía auditiva, debido a su alta sensibilidad y porque no requieren la cooperación del neonato. ${ }^{4}$

En 1994 el Comité Conjunto de Audición Infantil (JCIH) identificó algunos factores de riesgo específicos asociados con discapacidad auditiva, principalmente antecedentes de: infección intrauterina, familiares de hipoacusia neurosensorial hereditaria infantil, bajo peso al nacimiento, anomalías cráneo-faciales, meningoencefalitis bacteriana, exposición a medicamentos ototóxicos, hiperbilirrubinemia, puntaje de Apgar bajo, requerimiento de ventilación mecánica ${ }^{1,5}$ y prematuridad, pues durante el tercer trimestre del embarazo comienza la mielinización del nervio auditivo entre la cóclea y el tronco cerebral. ${ }^{4}$
La vía auditiva es una de las áreas más vulnerables del sistema nervioso central para neurotoxicidad por hiperbilirrubinemia; sin embargo, la correlación entre las concentraciones de bilirrubina y el desenlace auditivo permanece en estudio. Esto se debe a que la exposición a bilirrubina no puede cuantificarse con precisión y la toxicidad auditiva después de la exposición también es bastante variable, desde pérdida auditiva mínima hasta completa. ${ }^{6,7}$ El $80 \%$ de los neonatos prematuros que permanecen en la UCIN tienen hiperbilirrubinemia, que a menudo se manifiesta como ictericia. ${ }^{6}$ La bilirrubina es una sustancia neurotóxica que inhibe la producción de enzimas mitocondriales y la síntesis de ADN, con posterior bloqueo de la señalización excitadora de los nervios y daño de los núcleos cocleares. ${ }^{6}$

Saluja y colaboradores ${ }^{8}$ encontraron que de los neonatos con hiperbilirrubinemia que requirieron exanguinotransfusión, 46\% tuvieron alteración neuropática del sistema auditivo, concomitante con anormalidades en los potenciales evocados auditivos del tallo cerebral. ${ }^{8}$

Diversos estudios muestran resultados ambiguos en cuanto a la relación de factores de riesgo en pacientes prematuros y alteraciones de la vía auditiva. Wang y su grupo ${ }^{9}$ evaluaron a neonatos prematuros con igual o menos de 32 semanas de gestación, con la intención de analizar los potenciales evocados auditivos del tallo cerebral en pacientes con y sin factores de riesgo perina- 
tales asociados. Encontraron mayores auditivas, con prolongación de latencias interpico I-V, en pacientes con factores de riesgo perinatales y en los prematuros con bajo riesgo. El intervalo I-V aumentó ligeramente, sin diferencia estadísticamente significativa en comparación con neonatos de término. ${ }^{9}$

Con base en lo anterior, el objetivo de este estudio fue estimar la frecuencia de alteraciones auditivas en neonatos pretérmino a su egreso de la unidad de cuidados intensivos e identificar los factores de riesgo asociados con su manifestación.

\section{MATERIALES Y MÉTODOS}

Estudio transversal y retrospectivo, llevado a cabo en recién nacidos (menos de 37 semanas de gestación establecidas por fecha de última menstruación) en el Hospital Español de México, atendidos en la unidad de cuidados intensivos neonatales (UCIN) entre el 1 de enero de 2014 y el 31 de diciembre de 2016. La muestra se conformó mediante muestreo no probabilístico de casos consecutivos por cuota de tiempo. Criterios de exclusión: neonatos con malformaciones auditivas, síndromes genéticos, fallecidos durante su estancia en la UCIN (pues los potenciales evocados auditivos del tallo cerebral [PEATC] que se utilizaron para el estudio se efectuaron al egreso del paciente); pacientes enviados a otras unidades o a su domicilio, y si sucedió antes de las 35 semanas de gestación corregida (porque IOs PEATC se obtuvieron con semanas corregidas, mayores de 36, con la finalidad de evitar mayor inmadurez del sistema nervioso central al momento de efectuar la prueba y crear confusión o sesgos en los resultados); y quienes tuvieron expediente clínico incompleto. Se estudiaron las variables de sexo, semanas de gestación al nacimiento para conocer el grado de prematurez; restricción del crecimiento intrauterino, hiperbilirrubinemia, sepsis, hemorragia intraventricular y encefalopatía hipóxico-isquémica, además de antecedente de exposición a medicamentos potencialmente ototóxicos (furosemida y aminoglucósidos). A todos los neonatos se les valoraron los potenciales evocados auditivos del tallo cerebral (PEATC) 72 horas previas a su egreso.

De las variables incluidas, es importante mencionar definiciones o criterios usados para su medición:

Restricción del crecimiento intrauterino. Es el peso fetal por debajo del percentil 10 asociado con el índice de pulsatilidad del Doppler de la arteria umbilical por encima del percentil 95 para la edad gestacional. ${ }^{10}$

Hiperbilirrubinemia. En el paciente prematuro no existen cifras específicas de bilirrubina sérica para diagnosticar hiperbilirrubinemia y establecer el tratamiento, pues hay datos limitados para las recomendaciones basadas en evidencia. Por lo tanto, deben graficarse de acuerdo con el peso al nacimiento, las horas de vida extrauterina y las semanas de gestación, y con ello conocer el riesgo de complicaciones. En este estudio se utilizó el normograma de Bhutani para pacientes mayores de 35 semanas de gestación y la tabla sugerida por Watchko y Bhutani para menores de la misma edad, con la intención de estimar el riesgo y establecer el tratamiento. ${ }^{11,12}$

Sepsis. Haque ${ }^{13}$ definió los criterios para determinar la sepsis en neonatos. Esta variable se consideró al momento de su confirmación, de acuerdo con los signos clínicos de infección y hemocultivo positivo; y diagnóstico probable cuando no se aislaron patógenos, pero hubo sepsis clínica y al menos dos reportes de laboratorio alterados, indicando alta sospecha de sepsis. ${ }^{13}$

Hemorragia intraventricular. La hemorragia intraventricular se diagnosticó por ultrasonido transfontanelar al ingreso del paciente a la UCIN. Se analizaron y registraron: tipo de hemorragia intraventricular según la clasificación de Papile, 
desarrollada para estudios de tomografía; sin embargo, ese esquema puede aplicarse para ultrasonido transfontanelar. Grado 1: se refiere a la hemorragia subependimaria confinada a la matriz germinal; Grado 2: hemorragia en el lumen de ventrículos laterales sin dilatación de ventrículos; Grado 3: hemorragia en los ventrículos laterales asociada con dilatación de los ventrículos y Grado 4: daño al parénquima cerebral. ${ }^{14}$

Encefalopatía hipóxico-isquémica. Hasta el momento no existe una prueba específica para el diagnóstico certero de encefalopatía hipóxicoisquémica en el recién nacido; sin embargo, puede distinguirse de la encefalopatía neonatal por depresión del nivel de conciencia, a menudo con depresión respiratoria, anormalidad del tono muscular y convulsiones. La evidencia de puntaje bajo de Apgar y acidosis metabólica en sangre arterial del cordón umbilical, acompañados de disfunción neurológica, sugiere fuertemente lesión hipóxico-isquémica. La lesión concomitante con otros órganos, como el hígado con elevada concentración de transaminasas, los riñones con elevación de creatinina y el corazón con altas cifras de creatina cinasa-MB y troponina $\mathrm{T}$ proporcionan evidencia adicional de lesión hipóxico-isquémica. Además, el patrón de lesión en la resonancia magnética del cerebro puede confirmar con mayor certeza el diagnóstico; sin embargo, no siempre es factible realizarla por la inestabilidad del paciente y el riesgo que conlleva el traslado. ${ }^{15}$ Los recién nacidos con sospecha de encefalopatía hipóxico-isquémica se clasifican según el sistema de estadificación Sarnat, que evalúa el nivel de conciencia, tono muscular, reflejos tendinosos, reflejos complejos y función autónoma. Existen tres categorías: etapa I (leve), etapa II (moderada) y etapa III (severa). ${ }^{15}$

Potenciales evocados auditivos del tallo cerebral (PEATC). Estos se estimaron con un equipo marca Cadwell. La técnica consistió en colocar tres electrodos: uno en cada lóbulo auricular (A1/ A2) y otro en el vértice del cráneo Cz (sistema internacional 10-20 del EEG); posteriormente se estimuló la vía auditiva a través de un micrófono insertado en el canal auditivo. El estímulo consistió en "clics", con intensidad de $80 \mathrm{~dB}$ y frecuencia de $12.1 / \mathrm{s} .{ }^{1}$ Las señales obtenidas se promediaron a 1000 estímulos auditivos en 3 a 4 replicaciones, esto aseguró la reproducibilidad de los hallazgos. Los elementos obtenidos de estas estimulaciones consistieron en cinco ondas negativas en los primeros 8 milisegundos de haber iniciado el estímulo auditivo. Cada onda representa una estructura del sistema auditivo: la onda I es la respuesta del nervio acústico, la onda II corresponde al núcleo coclear, y las ondas III, IV y $\mathrm{V}$ representan los relevos del complejo olivar superior, puente y mesencéfalo (colículo inferior), respectivamente. Las ondas I, III y V son las más consistentes y, por tanto, las que se utilizan para evaluar la vía auditiva periférica y central. Entre las ondas se analizaron las latencias interpico I-III, III-V y I-V, o el tiempo de conducción auditiva central. Los estudios fueron interpretados por un neurofisiólogo certificado por el Consejo Mexicano de Neurofisiología Clínica.

Para el análisis de los datos se utilizó el programa estadístico SPSS versión 22.0. Se realizó estadística descriptiva de las variables, mediante frecuencias y proporciones; posteriormente se estableció la frecuencia de las alteraciones unilaterales y bilaterales para las latencias interpico I-III, III-V y I-V, y las ondas I, III y V. Para describir las diferencias entre los pacientes con alteración unilateral o bilateral se utilizó la prueba de $\chi^{2}$ con corrección de Yates (en caso necesario).

Debido a la cantidad insuficiente de pacientes con afectación unilateral, el análisis de riesgos se efectuó en los neonatos que tuvieron alteración uni o bilateral. Los análisis se realizaron exclusivamente para las latencias como señal de daño. Los riesgos se presentaron en razón de momios $(\mathrm{OR})$, con intervalos de confianza de 95\%. Se 
consideró estadísticamente significativo el valor de $p<0.05$. El protocolo fue aprobado por el Comité de Investigación y Ética del Hospital Español de México (EHE0051), según las recomendaciones de la Declaración de Helsinki. ${ }^{16}$

\section{RESULTADOS}

Se registraron 459 recién nacidos pretérmino y se excluyeron 95 por no cumplir con los criterios de selección, por lo que la población final la conformaron 364 neonatos: 193 (53\%) varones y $171(47 \%)$ mujeres. El promedio de semanas de gestación fue de 34 (límites de 24 a 36). Se encontraron $11(3 \%)$ prematuros extremos, 92 $(25.3 \%)$ muy prematuros y 261 (71.7\%) prematuros moderados, según la clasificación de la OMS.

Entre las enfermedades asociadas con alteraciones auditivas se encontraron: restricción del crecimiento intrauterino en 47 (12.9\%), hiperbilirrubinemia 181 (49.7\%), sepsis 113 (31\%), hemorragia intraventricular $13(3.6 \%)$ y encefalopatía hipóxico-isquémica en 5 (1.4\%) casos. El antecedente de exposición a furosemida se registró en $40(11 \%)$ pacientes y aminoglucósidos en 235 (64.6\%).

Respecto de los potenciales evocados auditivos del tallo cerebral (PEATC), se encontró que la alteración más frecuente fue la prolongación de la latencia interpico I-III en 138 (37.9\%) neonatos, seguida de la latencia interpico I-V en 49 (13.5\%). Los 16 (4.4\%) restantes tuvieron prolongación de la latencia interpico III-V. En cuanto a las ondas: la $\mathrm{V}$ resultó alterada en 38 $(10.4 \%)$ casos, la I en $24(6.6 \%)$ y la III en 7 (1.9\%). Cuadro 1

Para describir las diferencias entre pacientes con alteración unilateral o bilateral, el análisis bivariado reportó mayor prolongación de la latencia I-III para los neonatos con hiperbilirrubinemia $(p=.001)$ y sepsis $(p=.018)$; en la III-V para quienes tuvieron restricción del crecimiento intrauterino $(p=.005)$ y asociadas con hiperbilirrubinemia $(p=.004)$ y sepsis $(p=.006)$. En la latencia general I-V para los que tuvieron restricción del crecimiento intrauterino $(p=.015)$ $y$ asociadas con hiperbilirrubinemia $(p=.006)$ y hemorragia intraventricular $(p=.025)$. Cuadro 2

Cuadro 1. Características clínicas y potenciales evocados auditivos de tallo cerebral en neonatos atendidos en la UCIN $(\mathrm{n}=364)$ del Hospital Español (2014-2016)

\begin{tabular}{|c|c|c|c|}
\hline TC & Modalidad & Frecuencia & Proporción \\
\hline \multicolumn{4}{|c|}{ Característica } \\
\hline \multirow[t]{2}{*}{ Sexo } & Masculino & 193 & $(53 \%)$ \\
\hline & Femenino & 171 & $(47 \%)$ \\
\hline \multirow[t]{4}{*}{$\begin{array}{l}\text { Nivel de } \\
\text { prematurez }\end{array}$} & $\begin{array}{l}\text { Prematuros } \\
\text { extremos }\end{array}$ & 11 & $(3 \%)$ \\
\hline & $\begin{array}{c}\text { Muy } \\
\text { prematuros }\end{array}$ & 92 & $(25.3 \%)$ \\
\hline & $\begin{array}{l}\text { Prematuros } \\
\text { moderados }\end{array}$ & 261 & $(71.7 \%)$ \\
\hline & \multicolumn{3}{|c|}{ Enfermedades asociadas } \\
\hline RCIU & Sí & 47 & $(12.9 \%)$ \\
\hline $\begin{array}{l}\text { Hiperbilirrubi- } \\
\text { nemia }\end{array}$ & Sí & 181 & $(49.7 \%)$ \\
\hline Sepsis & Sí & 113 & $(31 \%)$ \\
\hline HIV & Sí & 13 & $(3.6 \%)$ \\
\hline $\mathrm{EHI}$ & Sí & 5 & $(1.4 \%)$ \\
\hline \multicolumn{4}{|c|}{ Medicamentos ototóxicos } \\
\hline Furosemida & Sí & 40 & $(11 \%)$ \\
\hline Aminoglucósidos & Sí & 235 & $(64.6 \%)$ \\
\hline \multicolumn{4}{|c|}{ PEATC } \\
\hline $\begin{array}{l}\text { Latencia } \\
\text { interpico I-III }\end{array}$ & Anormal & 138 & $(37.9 \%)$ \\
\hline $\begin{array}{l}\text { Latencia } \\
\text { interpico III-V }\end{array}$ & Anormal & 16 & $(4.4 \%)$ \\
\hline $\begin{array}{l}\text { Latencia } \\
\text { interpico I-V }\end{array}$ & Anormal & 49 & $(13.5 \%)$ \\
\hline Onda I & Anormal & 24 & $(6.6 \%)$ \\
\hline Onda III & Anormal & 7 & $(1.9 \%)$ \\
\hline Onda V & Anormal & 38 & $(10.4 \%)$ \\
\hline
\end{tabular}

PEATC: potenciales evocados auditivos de tallo cerebral; RCIU: restricción del crecimiento intrauterino; HIV: hemorragia intraventricular; EHI: encefalopatía hipóxico isquémica. 
Cuadro 2. Características clínicas según los reportes de los potenciales evocados auditivos de tallo cerebral $(\mathrm{n}=364)$

\begin{tabular}{|c|c|c|c|c|}
\hline \multirow{2}{*}{ Variables } & \multirow[t]{2}{*}{ Frecuencia } & \multirow{2}{*}{$\frac{\text { Alteración bilateral }}{\text { n (\%) }}$} & \multirow{2}{*}{$\begin{array}{c}\text { Alteración unilateral } \\
\mathbf{n}(\%)\end{array}$} & \multirow[t]{2}{*}{ p* } \\
\hline & & & & \\
\hline Latencia I-III & $\mathrm{n}=364$ & $\mathrm{n}=120$ & $\mathrm{n}=18$ & \\
\hline Género (masculino) & $193(53.0)$ & $57(47.5)$ & $13(72.2)$ & .116 \\
\hline Grado de prematuridad ** & $261(71.7)$ & $93(77.5)$ & $12(66.7)$ & .222 \\
\hline $\mathrm{RCIU} * * *$ & $47(12.9)$ & $15(12.5)$ & $4(22.2)$ & .535 \\
\hline Hiperbilirrubinemia & $181(49.7)$ & $85(70.8)$ & $15(83.3)$ & .001 \\
\hline Sepsis & $113(31.0)$ & $48(40.0)$ & $7(38.9)$ & .018 \\
\hline Hemorragia intraventricular & $13(3.6)$ & $5(4.2)$ & $2(11.1)$ & .265 \\
\hline Encefalopatía hipóxico-isquémica & $5(1.4)$ & $1(0.8)$ & $0(0.0)$ & .595 \\
\hline Furosemida & $40(11.0)$ & $12(10.0)$ & $3(16.7)$ & .724 \\
\hline Aminoglucósidos & $235(64.6)$ & $76(63.3)$ & $14(77.8)$ & .480 \\
\hline Latencia III-V & $\mathrm{n}=364$ & $\mathrm{n}=11$ & $\mathrm{n}=5$ & \\
\hline Género (masculino) & $193(53.0)$ & 7 (63.6) & $2(40.0)$ & .654 \\
\hline Grado de prematuridad** & $261(71.7)$ & $6(54.5)$ & $4(80.0)$ & .434 \\
\hline $\mathrm{RCIU} * * *$ & $47(12.9)$ & $4(36.4)$ & $3(60.0)$ & .005 \\
\hline Hiperbilirrubinemia & $181(49.7)$ & $10(90.9)$ & $4(80.0)$ & .004 \\
\hline Sepsis & $113(31.0)$ & $8(72.7)$ & $3(60.0)$ & .006 \\
\hline Hemorragia intraventricular & $13(3.6)$ & $0(0.0)$ & $0(0.0)$ & .551 \\
\hline Encefalopatía hipóxico-isquémica & $5(1.4)$ & $0(0.0)$ & $0(0.0)$ & .797 \\
\hline Furosemida & $40(11.0)$ & $1(9.1)$ & $1(20.0)$ & .824 \\
\hline Aminoglucósidos & $235(64.6)$ & $6(54.5)$ & $5(100.0)$ & .089 \\
\hline Latencia I-V & $\mathrm{n}=364$ & $\mathrm{n}=34$ & $\mathrm{n}=15$ & \\
\hline Género (femenino) & $171(47.0)$ & $19(55.9)$ & $7(46.7)$ & .550 \\
\hline Grado de prematuridad** & $261(71.7)$ & $22(64.7)$ & $10(66.7)$ & .571 \\
\hline $\mathrm{RCIU} * * *$ & $47(12.9)$ & $6(17.6)$ & $6(40.0)$ & .015 \\
\hline Hiperbilirrubinemia & $181(49.7)$ & $22(80.0)$ & $12(80.0)$ & .006 \\
\hline Sepsis & $113(31.0)$ & $15(44.1)$ & $5(33.3)$ & .231 \\
\hline Hemorragia intraventricular & $13(3.6)$ & $2(5.9)$ & $3(20.0)$ & .025 \\
\hline Encefalopatía hipóxico-isquémica & $5(1.4)$ & $1(2.9)$ & $0(0.0)$ & .636 \\
\hline Furosemida & $40(11.0)$ & $7(20.6)$ & $3(20.0)$ & .112 \\
\hline Aminoglucósidos & $235(64.6)$ & $19(55.9)$ & $11(73.3)$ & .436 \\
\hline
\end{tabular}

* p: significancia estadística por de $\chi^{2} ; * *$ prematuro moderado; $* * *$ RCIU: restricción del crecimiento intrauterino. 
El Cuadro 3 muestra la relación entre anormalidades en las latencias interpico con afectación uni o bilateral y las características clínicas estudiadas, considerando que pueden ser factores de riesgo asociados con las alteraciones auditivas de los neonatos. Para la latencia interpico I-III prolongada, registrada en 138 (37.9\%) pacientes, se encontraron como factores de riesgo asociados hiperbilirrubinemia: $\mathrm{OR}=4.71(2.97-7.48), \mathrm{p}=$ 0.001 y sepsis: $\mathrm{OR}=1.92(1.22-3.02), \mathrm{p}=0.005$. Para la prolongación en la latencia interpico III-V, encontrada en 16 neonatos (4.4\%), fueron hiperbilirrubinemia: $\mathrm{OR}=7.59$ (1.69-33.88), $\mathrm{p}$ $=0.002$ y sepsis: $\mathrm{OR}=5.31(1.80-15.66), \mathrm{p}=$ 0.002; también se encontró que la restricción del crecimiento intrauterino fue un factor protector: $\mathrm{OR}=0.191$ (0.07-0.49), $\mathrm{p}=.002$. Por último, en la prolongación de la latencia interpico global

Cuadro 3. Asociación entre anormalidades en las latencias auditivas y las características clínicas ( $\mathrm{n}=364)$ (continúa la siguiente columna)

\begin{tabular}{|c|c|c|}
\hline Variables & OR $(\operatorname{IC} 95 \%) *$ & $\mathbf{p}^{*}$ \\
\hline \multicolumn{3}{|c|}{ Latencia alterada (uni o bilateral) I-III } \\
\hline Género & $1.160(.759-1.773)$ & .493 \\
\hline $\begin{array}{l}\text { Grado de } \\
\text { prematuridad*** }\end{array}$ & $1.428(.882-2.312)$ & .147 \\
\hline $\mathrm{RCIU} * * * *$ & $1.129(.604-2.110)$ & .704 \\
\hline Hiperbilirrubinemia & $4.711(2.968-7.477)$ & .001 \\
\hline Sepsis & $1.919(1.220-3.019)$ & .005 \\
\hline $\begin{array}{l}\text { Hemorragia } \\
\text { intraventricular }\end{array}$ & $1.959(.645-5.955)$ & .236 \\
\hline $\begin{array}{l}\text { Encefalopatía } \\
\text { hipóxico-isquémica }\end{array}$ & $.405(.045-3.662)$ & .384 \\
\hline Furosemida & $.980(.498-1.932)$ & .955 \\
\hline Aminoglucósidos & $1.047(.672-1.632)$ & .838 \\
\hline \multicolumn{3}{|c|}{ Latencia alterada (uni o bilateral) III-V } \\
\hline Género & $1.139(.434-2.993)$ & .791 \\
\hline $\begin{array}{l}\text { Grado de } \\
\text { prematuridad*** }^{* *}\end{array}$ & $1.520(.567-4.076)$ & .416 \\
\hline $\mathrm{RCIU} * * * *$ & $.191(.075-.488)$ & .002 \\
\hline Hiperbilirrubinemia & 7.587 (1.699-33.881) & .002 \\
\hline
\end{tabular}

I-V, registrada en 49 (13.5\%) casos, se encontró como factor de riesgo hiperbilirrubinemia: OR $=2.59$ (1.36-4.95), $\mathrm{p}=0.003$; hemorragia intraventricular: $\mathrm{OR}=4.36(1.37-13.93), \mathrm{p}=0.021$ y prescripción de furosemida: $\mathrm{OR}=2.44$ (1.115.37), $\mathrm{p}=0.023$; como factor protector se reportó la restricción del crecimiento intrauterino: $\mathrm{OR}=$ $0.46(0.26-0.81), p=0.009$.

\section{DISCUSIÓN}

Diversos autores señalan que hay disfunción de la vía auditiva en 2 a $4 \%$ de los neonatos que egresan de la UCIN, incluso de $10 \%$ en prematuros. ${ }^{1,17}$ En este estudio, la prevalencia fue de $13.4 \%$ en la latencia global, posiblemente por los criterios de selección de la muestra; además, las alteraciones encontradas no fueron pérdida

\begin{tabular}{|c|c|c|}
\hline Variables & OR $(\operatorname{IC} 95 \%)^{*}$ & $\mathbf{p}^{*}$ \\
\hline Sepsis & $5.306(1.798-15.656)$ & .002 \\
\hline $\begin{array}{l}\text { Hemorragia } \\
\text { intraventricular }\end{array}$ & $.954(.933-.976)$ & .275 \\
\hline $\begin{array}{l}\text { Encefalopatía } \\
\text { hipóxico-isquémica }\end{array}$ & $.955(.934-.977)$ & .501 \\
\hline Furosemide & $1.165(.255-5.325)$ & .846 \\
\hline Aminoglucósidos & $1.218(.414-3.585)$ & .720 \\
\hline \multicolumn{3}{|c|}{ Latencia alterada (uni o bilateral) I-V } \\
\hline Género & $1.325(.725-2.423)$ & .359 \\
\hline $\begin{array}{l}\text { Grado de } \\
\text { prematuridad*** }\end{array}$ & $.707(.373-1.338)$ & .285 \\
\hline $\operatorname{RICU}^{* * * *}$ & $.457(.257-.812)$ & .009 \\
\hline Hiperbilirrubinemia & $2.590(1.357-4.945)$ & .003 \\
\hline Sepsis & $1.646(.886-3.057)$ & .112 \\
\hline $\begin{array}{l}\text { Hemorragia } \\
\text { intraventricular }\end{array}$ & $4.361(1.365-13.927)$ & .021 \\
\hline $\begin{array}{l}\text { Encefalopatía } \\
\text { hipóxico-isquémica }\end{array}$ & $1.620(.177-14.799)$ & .684 \\
\hline Furosemida & $2.436(1.105-5.367)$ & .023 \\
\hline Aminoglucósidos & $.847(.456-1.574)$ & .600 \\
\hline
\end{tabular}

* OR: Razón de momios, IC95\%: Intervalo de confianza de $95 \%$; ** p: significación estadística; ${ }^{* * *}$ prematuro moderado, **** RCIU: restricción del crecimiento intrauterino. 
de audición, sino defectos en la conducción de la vía auditiva.

Además de los factores de riesgo específicos asociados con discapacidad auditiva descritos por el Comité Conjunto de Audición Infantil $(\mathrm{JClH}),{ }^{1,5}$ diversos estudios han reportado: semanas de gestación al nacimiento, ${ }^{1}$ restricción del crecimiento intrauterino, ${ }^{4}$ hiperbilirrubine$\mathrm{mia}^{1,6,7,8,18}$ sepsis, hemorragia intraventricular, ${ }^{19}$ encefalopatía hipóxico-isquémica, entre otros. ${ }^{20}$ Estas variables coinciden con lo encontrado en nuestro estudio, en relación con la hiperbilirrubinemia, sepsis, Hemorragia intraventricular y toxicidad por furosemida, que resultaron factores de riesgo; por su parte, la restricción del crecimiento intrauterino fue un factor de protección de alteraciones auditivas.

Agrisani y sus colaboradores ${ }^{21}$ reportaron alteraciones en los potenciales evocados auditivos del tallo cerebral de recién nacidos de término de género masculino. Aunque no propusieron alguna explicación de la fisiopatogenia de estos hallazgos, ${ }^{21}$ en nuestro estudio encontramos mayor frecuencia en la prolongación de las latencias interpico I-III y III-V en el sexo masculino vs I-V del femenino, sin diferencias estadísticamente significativas entre ambos géneros.

La hemorragia intraventricular es otro factor de riesgo relacionado con daño auditivo. ${ }^{1,19}$ Diversos estudios señalan que los neonatos con hemorragia intraventricular grado I y II pueden tener anomalías neurológicas (retraso del desarrollo cognitivo y discapacidad auditiva) comparados con los prematuros y quienes tienen reportes normales en el ultrasonido transfontanelar. ${ }^{19} \mathrm{El} 7.4 \%$ de los pacientes con esta alteración tiene cierto grado de deficiencia auditiva, ${ }^{22}$ debido al deterioro de la matriz germinal, una región altamente vascularizada ubicada entre el núcleo caudado y el tálamo, de donde surgen células gliales y neuronas responsables de la mielini- zación y el desarrollo cortical y subcortical. Esto evidencia la importancia de la evaluación audiológica temprana en los pacientes. ${ }^{23}$ En nuestro estudio, la hemorragia interventricular fue un factor de riesgo con significación estadística de $p=0.21$, con una prolongación de latencia interpico I-V, lo que sugiere un defecto de conducción central de la audición entre el nervio acústico y mesencéfalo.

Otro factor asociado es la hiperbilirrubinemia, principalmente en pacientes con elevadas concentraciones de bilirrubina no conjugada, porque puede provocar encefalopatía por Kernícterus o requerimiento de exanguinotransfusión, debido al daño del núcleo coclear. ${ }^{6,7,18}$ Esta alteración existió en la mitad de los neonatos y resultó ser un factor de riesgo, aunque las concentraciones no se reportaran en límites que ameritaran exanguinotransfusión, por lo que solo requirieron fototerapia.

Las infecciones son comunes en los neonatos prematuros, debido al sistema inmunitario poco desarrollado y que pueden convertirse rápidamente en una amenaza para la vida. Los aminoglucósidos son el tratamiento de primera línea para la sospecha clínica o septicemia comprobada. Su alta eficacia, bajo grado de resistencia y costo accesible hacen de este grupo de fármacos una opción efectiva y recomendada; sin embargo, debido a la nefro y ototoxicidad conocidas se sugiere el control regular de las concentraciones en sangre. ${ }^{24}$ En este estudio, 65\% de los pacientes requirió aminoglucósidos a su ingreso a la UCIN; no obstante, nuestros hallazgos no mostraron asociación entre su prescripción y alteraciones en los potenciales evocados auditivos del tallo cerebral. La bibliografía evidencia asociación entre ambas variables, por lo que a futuro puede ampliarse el estudio y evaluar si existe significación estadística.

La sepsis fue otra variable que apareció en una tercera parte de la muestra, quizá relacionada 
con alguna alteración auditiva por la prescripción de aminoglucósidos como tratamiento inicial. Sin embargo, no hubo asociación entre esta variable y las alteraciones de los PEATC, pero sí con la manifestación de sepsis.

Una práctica común en pacientes prematuros de la UCIN es la indicación de diuréticos, que reducen el exceso de líquido pulmonar, mejoran el intercambio de gases y disminuyen la necesidad de asistencia respiratoria. ${ }^{25}$ Aproximadamente $40 \%$ de los prematuros están expuestos, al menos, a un diurético durante su estancia en la $\mathrm{UCIN}$, principalmente furosemida. ${ }^{26}$ Aunque actualmente se desconoce el mecanismo preciso de posible ototoxicidad inducida por furosemida, se piensa que el fármaco puede influir en la función coclear al cambiar la composición iónica del líquido endolinfático y alterar el potencial eléctrico endococlear. ${ }^{27}$ En este estudio se encontró asociación entre furosemida y alteraciones auditivas en la latencia interpico I-V.

La restricción del crecimiento intrauterino es otro factor relacionado con prematuridad y alteraciones auditivas en neonatos. ${ }^{4}$ En esta investigación se encontró en $13 \%$ del total de la muestra. En las alteraciones uni y bilaterales para las latencias interpico III-V y I-V se observó que previene al neonato de alteraciones auditivas a su egreso de la UCIN, quizá porque durante el embarazo se prescriben esteroides sistémicos a las madres, para maduración del feto, lo que puede acelerar el proceso de mielinización central; sin embargo, esta consideración es meramente especulativa.

Este estudio es la primera etapa de una serie de trabajos de seguimiento de pacientes prematuros con alteraciones en los potenciales evocados auditivos del tallo cerebral. En esta primera etapa solo se describe la frecuencia de alteraciones y su asociación con diferentes enfermedades e indicación de medicamentos.
Entre las limitantes de la investigación se citan: 1) falta de estudio de seguimiento neurológico a largo plazo; por lo tanto, se desconoce la relación entre alteraciones de los potenciales evocados auditivos del tallo cerebral y sus consecuencias neurológicas a posteriori. Este aspecto será motivo de estudio ulterior, en el que se harán valoraciones a los seis meses y al año para determinar el significado clínico en el desarrollo neurológico de los pacientes, además de determinar si las alteraciones auditivas encontradas disminuyeron conforme a la maduración en los mecanismos de mielinización; 2) la población de estudio con afectación unilateral fue muy pequeña, lo que imposibilita hacer una generalización de los resultados y extrapolarlos a otras poblaciones; y 3) el diseño con el que se realizó el estudio e impidió describir el efecto de los factores de riesgo en el neurodesarrollo de los pacientes.

\section{CONCLUSIONES}

La hiperbilirrubinemia, sepsis, hemorragia intraventricular e indicación de furosemida son factores que incrementan el riesgo de defectos en la conducción auditiva, principalmente en las latencias interpico I-III, III-V y I-V. La restricción del crecimiento intrauterino es un factor protector de alteraciones auditivas centrales.

\section{REFERENCIAS}

1. Huang $L$, et al. An analysis of hearing screening test results in 2291 premature infants of Chinese population. Int J Pediatr Otorhinolaryngol 2017;95:15-19. doi: 10.1016/j. ijporl.2017.01.027

2. Maggiolo LM, et al. Dificultades de lenguaje en niños preescolares con antecedente de prematuridad extrema. Rev Chil Pediatr 2014;85(3):319-327. doi:10.4067/s037041062014000300008

3. Wien MA, et al. The association among prematurity, cochlear hyperintensity, and hearing loss. Neuroradiol J 2017;30(5):448-453. doi: 10.1177/1971400917709623

4. Seethapathy J, et al. Auditory brainstem response in very preterm, moderately preterm and late preterm infants. Int J Pediatr Otorhinolaryngol 2018;111:119-127. doi:10.1016/j. ijporl.2018.06.006 
5. Nuñez-Batalla F, et al. Indicadores de riesgo de hipoacusia neurosensorial infantil. Acta Otorrinolaringológica Española 2012;63(5): 382-390.

6. Gi-Sung $\mathrm{N}$, et al. Hyperbilirubinemia and follow-up auditory brainstem responses in preterm infants. Clin Exp Otorhinolaryngol 2019;12(2):163-168. doi: 10.21053/ ceo.2018.00899.

7. Shapiro $S M$, et al. Bilirubin and the auditory system. J Perinatol 2001;21(S1):S52-S55. doi: 10.1038/sj.jp.7210635

8. Saluja, et al. Auditory neuropathy spectrum disorder in late preterm and term infants with severe jaundice. Int J Pediatr Otorhinolaryngol 2010;74(11):1292-1297. doi: 10.1016/j.ijporl.2010.08.007

9. Wang $C$, et al. Brainstem auditory response findings in very preterm babies in the intensive care unit. Neonatology 2015;107(2):157-160. doi: 10.1159/000368957

10. Cabero-I-Roura L, et al. Restricción del crecimiento intrauterino en Protocolos de Medicina Materno-fetal (Perinatologia). 4a Edición. Barcelona: Ergon; 2014.

11. Maisels MJ, et al. An approach to the management of hyperbilirubinemia in the preterm infant less than 35 weeks of gestation. J Perinatol 2012;32(9):660-664. doi:10.1038/ jp.2012.71

12. American Academy of Pediatrics Subcommittee on Hyperbilirubinemia. Management of hyperbilirubinemia in the newborn infant 35 or more weeks of gestation. Pediatrics 2004;114(1):297-316. doi:10.1542/peds.114.1.297

13. Haque KN. Definitions of bloodstream infection in the newborn. Pediatr Crit Care Med 2005;(Suppl 6):S45-49. doi:10.1097/01.pcc.0000161946.73305.0a

14. Papile LA, et al. Incidence and evolution of subependymal and intraventricular hemorrhage: A study of infants with birth weights less than 1,500 gm. J Pediatr 1978;92(4):52934. doi:10.1016/s0022-3476(78)80282-0

15. Douglas-Escobar $M$, et al. Hypoxic-ischemic encephalopathy: a review for the clinician. JAMA Pediatr 2015;169(4):397-403. doi:10.1001/jamapediatrics.2014.3269

16. Asociación Médica Mundial. Declaración de Helsinki de la AMM - Principios éticos para las investigaciones médicas en seres humanos. Marzo, 2017.
17. Stipdonk LW, et al. Auditory brainstem maturation in normal-hearing infants born preterm: a meta-analysis. Dev Med Child Neurol 2016;58(10):1009-1015. doi: 10.1111/ dmon.13151

18. Ahlfors $\mathrm{CE}$, et al. Unbound bilirubin concentration is associated with abnormal automated auditory brainstem response for jaundice newborns. Pediatrics 2008;121(5):976-8.

19. Da Silva LS, et al. The effect of peri-intraventricular hemorrhage on the auditory pathway of infants. Int J Pediatr Otorhinolaryngol 2018;112:24-26. doi: 10.1016/j. ijporl.2018.06.026

20. Fitzgerald MP, et al. Hearing impairment and hypoxia ischaemic encephalopathy: Incidence and associated factors. Eur J Paediatr Neurol 2019;23(1):81-6. doi: 10.1016/j. ejpn.2018.10.002.

21. Angrisani RM, et al. Electrophysiological characterization of hearing in small for gestational age premature infants. CoDAS 2013;25(1):22-8.

22. Tiensoli LO, et al. Hearing screening in a public hospital in Belo Horizonte, Minas Gerais State, Brazil: hearing impairment and risk factors in neonates and infants. Cad Saude Publica 2007;23:1431-1441.

23. Bolisetty $\mathrm{S}$, et al. Intraventricular hemorrhage and neurodevelopmental outcomes in extreme preterm infants. Pediatrics 2014;133:55-62.

24. Bitner-Glindzicz $M$, et al. Gentamicin, genetic variation and deafness in preterm children. BMC Pediatr 2014;14(1):66. doi: 10.1186/1471-2431-14-66.

25. Wang $L A$, et al. Prolonged furosemide exposure and risk of abonormal newborn hearing screen in premature infants. Early Hum Dev 2018;125:26-30. doi:10.1016/j.earlhumdev.2018.08.009

26. Chantala K, et al. Diuretic exposure in premature infants from 1997 to 2011. Am J Perinatol 2014;32(1):4956. doi:10.1055/s-0034-1373845.

27. Rybak LP, et al. Effect of furosemide upon endolymph potassium concentration. Hear Res 1982;7(2):223-31. doi:10.1016/0378-5955(82)90015-6 